\title{
A Conversation with Aaron Straight
}

\author{
INTERVIEWER: KIM BAUMANN \\ Chief Editor, Nature Reviews Molecular Cell Biology
}

Aaron Straight is an Associate Professor of Biochemistry at Stanford University.

\begin{abstract}
Kim Baumann: Your laboratory works on different aspects of chromosome biology that are related to understanding how the segregation of chromosomes occurs in a correct way. This includes work on the centromere and how it drives assembly of kinetochores, as well as on RNA biology and how RNAs regulate the structure and organization of chromosomes. Could you start by telling us about the connections between these two fields?
\end{abstract}

Dr. Straight: Those can seem like somewhat disparate fields and not necessarily connected. I started out trying to understand chromosome segregation because I was interested in the mechanics of chromosome movement and how and what controls the dynamic movement of chromosomes during anaphase and spindle elongation. Those problems are still interesting problems, but in some ways, I feel like we have gotten away from that because something else caught my attention.

A really fascinating problem in cell biology that will take decades to play out is that both of those examples that you gave - which are near and dear to my heart—are not so much about the specific mechanics of moving chromosomes and controlling the regulation of when they separate, but more about how a cell sets up specific functional domains of chromosomes, and in the face of all the other cellular processes-whether that is cellular mitosis or differentiation - how it decides how to specify a particular function and then attempt to reinforce that function over time, whether it's on a short timescale like a cell cycle, or the lifetime of an organism. For the most part, what I work on is chromosome-based functionalization. The centromere is a great example of that because it's made of DNA and histones and chromosome proteins, but it has a very unique function that's distinct from the content of the other parts of the chromosome. As far as we know that function is, at least in humans, primarily encoded not at the DNA sequence level but by the proteins that associate there.

Kim Baumann: And it's a function that needs to be maintained spatially in the chromosome. It's a region of the chromosome.

Dr. Straight: That gets right to the heart of it. If it's within some part of the chromosome but it's not the sequence defining it, then what gives it boundaries and what gives it identity? As the cells go through division, if the cell is duplicating and diluting it every time it replicates its DNA and chromatin, how is it maintained or reassessed? We spent several years trying to understand that problem. Really, the core of that problem for the centromeres is that there's a specific histone that's distinct from the rest of the histones, so there must be some cellular mechanism that can recognize that and say, "This is where we put more of that identifying histone and we don't put it elsewhere and we don't put other things here that shouldn't be here." Even though that sounds trivial, it turns out to be quite a complicated problem to solve. We are starting to touch on the machinery that does that sort of recognition of what a chromosome does, but I think we are far from understanding how you couple that recognition to the reassembly and the protection of that part of the chromosome from the rest of the chromosome.

Kim Baumann: How does RNA fit into this?

Dr. Straight: We're very new to that world. We've really been working on that problem seriously for maybe four or five years. We fell into that almost because of an accidental study of the centromere region. We were interested in a general concept, which is that there are certain RNAs that are well established that bind chromosomes and change their function. The best known is female dosage compensation: One X chromosome gets coated by RNA and silenced and the other doesn't. So we asked the generic question: Are there other RNAs that might act in that kind of mechanism to control the chromosome fate or chromosome structure?

Kim Baumann: Do you mean specifically heterochromatic regions, or more than that?

Dr. Straight: I wish we'd been that well-informed when we started, but I don't think we had thought through the problem very well at that point, so we just took an unbiased look: What RNAs stick to chromosomes? But we did that in mitosis when transcription is, for the most part, repressed. This is in human cells. We jump between a lot of different organisms in the lab, but this RNA stuff has been in human cells, although we are now moving in

(C) 2017 Straight. This article is distributed under the terms of the Creative Commons Attribution-NonCommercial License, which permits reuse and redistribution, except for commercial purposes, provided that the original author and source are credited. 
to other systems. The idea was: Can we take an unbiased look? Can we just essentially take a census or a visual assay to ask where do RNAs bind on human chromosomes? We chose mitotic chromosomes because transcription's shut down for the most part. We developed a labeling system to look at it, and we saw something pretty extraordinary, which was that the RNAs stayed associated with mitotic chromosomes. In particular, they concentrated around centromeres. We didn't anticipate that would be the case, but we knew how to work with that part of the chromosome so that helped us get more interested in the problem. We've spent a few years now working on that.

Kim Baumann: These RNAs are transcribed from those regions and then they remain there? How does that work?

Dr. Straight: That is an extremely good question, because they are exactly as you just described it. They are RNAs that are transcribed from those regions, but maintain their associations with those regions. We've recently discovered that those RNAs are involved in bringing a series of histone-modifying enzymes to that locus to actually modify the chromatin and help repress it. That's how it comes back to the centromere, because these are, in some sense, nonsequence-dependent events. We don't think the RNA sequence is important.

Kim Baumann: Is there any specific structure to the RNAs? Are there characteristics of these RNAs that make them work the way they work?

Dr. Straight: We've tried very hard to understand that. We've even done some specific structure mapping on the RNAs. We really have no evidence that there is a specific structure involved. Instead, we are thinking that the RNAs are acting as a platform just to increase the local concentration of the enzymes that bind them.

Kim Baumann: So it's mostly a physical function?

Dr. Straight: We think so.

Kim Baumann: Do they also regulate the activity of the enzymes, or do they just bring them?

Dr. Straight: We have tested that, and our data so far is that there's not specific regulation of the enzymes. Rather than changing the activity, they essentially localize and concentrate the activity. So, it's a way of redistributing the activity to a particular site - as opposed to modifying an activity - so it acts in one place and not another.

Coming back to that larger problem: How these are related? I think both of those problems are problems in how you maintain an epigenetically defined locus. I would say the RNA project that we've started to work on is really still in its infancy. I think that's a general problem for people studying noncoding RNAs in any organism, in that now there are tens of thousands identified, and we know the functions of a handful.

Kim Baumann: Do you know anything about how they are activated? Are they constitutively active because these regions are defined and they remain there? This is a region that is maintained. Are they being constantly produced?
Dr. Straight: We've looked at that, and we know that they're being synthesized in one particular part of the cell cycle, in $\mathrm{G}_{2}$, before the cell goes into mitosis. Maybe that's just to make sure that the transcript is there so that, when the chromosomes condense, it's at the right place at the right time. We don't know what the regulation of their synthesis is. We just haven't done those experiments. That's an interesting problem: whether there is regulated behavior. For instance, if you go to an early embryo or pluripotent cell, they lose a lot of that heterochromatin. There has to be some regulation of that domain, whether it's through RNA or whether it's through something else.

Kim Baumann: Do they function also in other parts of the chromosome, or are they specific to the centromere?

Dr. Straight: They're not specific to the centromere.

Kim Baumann: So it's maintaining, or perhaps defining, different regions?

Dr. Straight: We think for this particular class of these modifying enzymes that it is really involved in repressing all kinds of different repeat sequences in the human genome, whether those are centromeric, transposable elements, or endogenous retroviruses. Whereas, we don't think these RNAs affect the euchromatic genome very much. We think this process is quite specific to making heterochromatin and keeping it.

Kim Baumann: What is the next step in studying these?

Dr. Straight: We realized we were studying one RNA. We just had this one thing we're studying, and that's essentially how the field has been going. You'll find an RNA that's interesting and you'll bang away on it for a while and learn something about the biology about that one RNA. But what we wanted to do is ask, can we develop a method that allows us to look at all the RNAs? Can we globally find all the RNAs that bind to human chromosomes, and then understand where they are acting and maybe try to understand how they function? So just over the past two years, we've developed a method called chromosome-associated RNA sequencing, that allows us to essentially link an RNA to the site where it's bound to the DNA through a direct linkage. It's a physical link between the RNA and DNA. That's advantageous because it doesn't matter if it's coming from the site where it is transcribed or if it's going to some other part of the chromosome and acting, as long as we can capture that interaction. We've just recently done that and shown that we can, using highthroughput sequencing, identify all the other RNAs and all of the sites where they are bound. By doing that you immediately begin to generate hypotheses about how an RNA might bind.

For instance, we developed this technique in Drosophila because the genome is a bit smaller so it is easier to do, and the first thing that jumped out at us is that the dosage compensation RNAs, which control the expression of the $\mathrm{X}$ and $\mathrm{Y}$ balance, just beautifully coat the entire $\mathrm{X}$ chromosome. So we know that we can detect cis interactions for those RNAs. But we also see those RNAs going 
to other parts of the genome. And we see a lot of other RNAs with interesting behaviors. I feel like this is just beginning to scratch the surface.

Kim Baumann: It will be interesting to see how they are targeted against certain regions. It's a chicken-and-egg thing, because they target factors to the chromatin and vice versa, but if they are not just where they are transcribed, they need to reach their destination.

Dr. Straight: That is one the most important questions in the field right now. For noncoding RNAs, most of them we do not understand how they're targeted to where they act, and we don't understand how they are maintained there. We know that RNAs can bind to different complexes and RNA-binding proteins, but what gives them selectivity for a place in the genome is really a black box. I don't think that's a problem that is going to be solved by one lab. That's a problem for the whole field to try and understand.

Kim Baumann: There can be mechanisms that involve sequence-specific and nonsequence-specific events, right? With many factors, perhaps?

Dr. Straight: There are good examples of sequence-specific and nonsequence-specific factors that are involved in the process, but I think we still don't understand the specificity problem. If it is sequence-specific, we do understand it to some extent. For the ones that aren't, that's a much tougher problem, but it's fun to think about.

Kim Baumann: Going back to the RNAs that function in the centromeric area, how are they kept there? You say that there is no sequence-specificity, so what does it tell you about how that region functions? If you were to disrupt any of those mechanisms, what happens?
Dr. Straight: The poster child to really understand these problems is studies in fission yeast. That, arguably, is where mechanisms for RNAs controlling chromatin have been best worked out in these regions around centromeres. In that case though, it's known that there are sequence-dependent interactions that help control shutting down the chromatin, and if you destroy that system in fission yeast it causes chromosome desegregation.

Kim Baumann: The whole machinery is disrupted?

Dr. Straight: Exactly. We've just recently been able to test that in humans, and because now you can do things like modify genes with CRISPR and actually make mutants in the genome like you would in yeast, and you can do those experiments now. We find that when we compromise that RNA-dependent system, we start to derepress all of these heterochromatic regions that depend on our repeat sequence. That's part of why we think one of the key features of these systems is to ensure that you maintain the repression of heterochromatic regions, which is a bit chicken-andegg because it requires transcription in order to repress the transcription that's occurring there. Understanding how that balance plays out, whether that's a way for the cell to be able to regulate the relative expression from some of these regions, is also an interesting problem to think about.

Kim Baumann: Is this going to be a major focus for your lab for now?

Dr. Straight: We will keep pushing on this for a while. We haven't abandoned the centromere yet. I love that part of the chromosome and we'll keep working there. I think this represents maybe a broader class of problems, and I'm hopeful that we'll get some interesting insight. 


\section{$\$_{\text {CSH\& }}^{\infty}$ Cold Spring Harbor Symposia SYMPOSIA On Quantitative Biology}

\section{A Conversation with Aaron Straight}

Cold Spring Harb Symp Quant Biol 2017 82: 413-415 originally published online April 26, 2018 Access the most recent version at doi:10.1101/sqb.2017.82.035022

Creative This article is distributed under the terms of the

Commons http://creativecommons.org/licenses/by-nc/4.0/, which permits reuse and

License redistribution, except for commercial purposes, provided that the original author and source are credited.

Email Alerting Receive free email alerts when new articles cite this article - sign up in Service the box at the top right corner of the article or click here. 